\title{
Graphing Laboratory Data in Microsoft Windows
}

\author{
Glenn S. Kohne \\ Loyola College in Maryland
}

\begin{abstract}
As the volume of Windows applications multiplies and their sophistication increases, each application tends to do more functions thereby making its installation, configuration, and use more complex. There is a population of laboratory instructors who would like to make available to their students some very specific data handling programs that would be simple to install, configure, and use. This paper introduces and makes available two such data capture and graphing programs. The first program, "Curvefit", accepts experimental data points and fits an ndegree polynomial ( $n=1$ to 5 ). It provides very easy to use graphing mechanisms for the screen and for the printer. It has been adopted for course use by one of our materials science professors. The second program, "Diode", provides the same experimental data collection and graphing services as the first but it fits an exponential curve through the data points. This program is intended to provide the exponential equation describing a semiconductor diode. This program is used in the basic electronics course and associated laboratory at Loyola College.
\end{abstract}

\section{Introduction}

There is a wide variety of software designed to provide mathematical analysis and graphing functions to engineering faculty and students. The most successful (commercially) of these provide a wide variety of very powerful mathematical tools at a very reasonable price. In many undergraduate (especially freshman) laboratory courses, the time required for the students to learn to use the data collection, equation fitting and graph configuring functions of these tools can exceed the time devoted to the laboratory's experiments.

The two Microsoft Windows application programs presented here are simple to install and to use. The graphic user interface (GUI) provides direct and intuitive access to data collection, equation fitting and graph configuration operations. Average students can be expected to fully understand the use of these application programs in under ten minutes. This will encourage the students to experiment with the various curves that can be fitted as well as to try different specifications for the graphic presentation of their data.

\section{Description \& Operation}

The two applications appear quite similar to the user. The Principal functions are launched by a menu selection. The menu for "Curvefit" is shown below. 


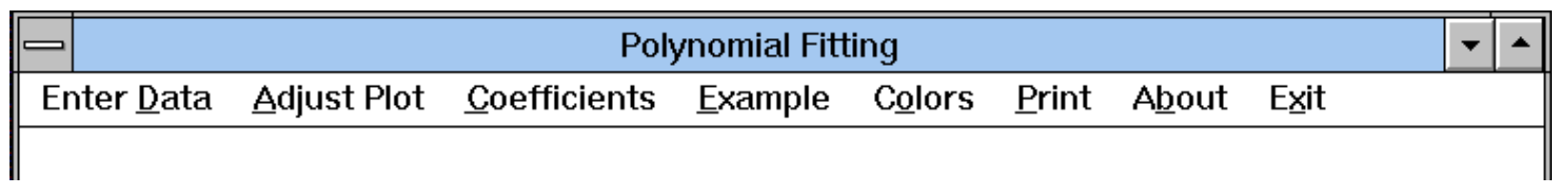

The Enter Data menu spawns the data collection screen shown below. It can be seen that entry and editing of data is both intuitive and obvious. Additional operations available on this screen are the saving of entered data to a disk file and the loading of previously saved data on disk. The degree of the polynomial to be fitted is specified here.

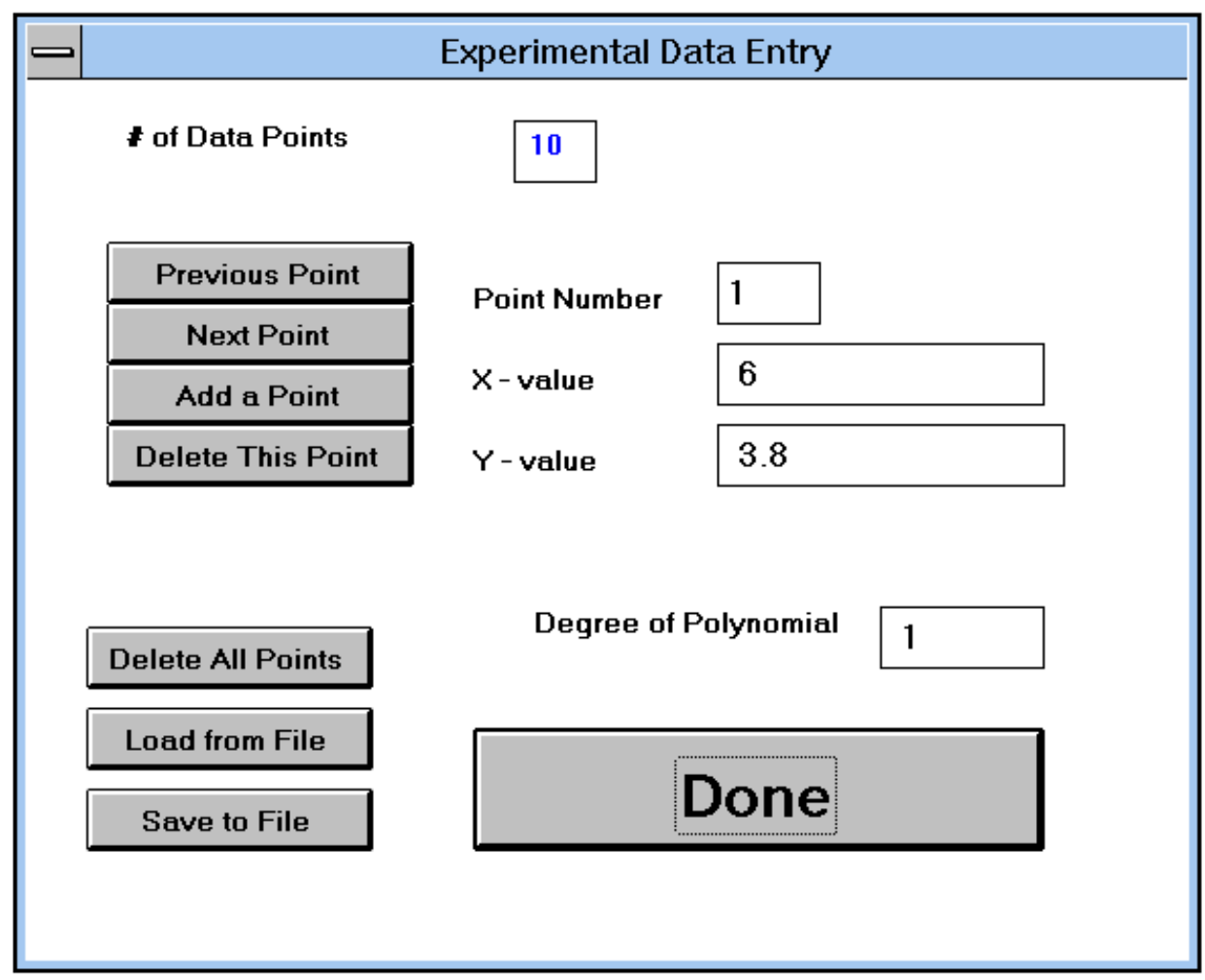

The Adjust Plot menu selection spawns the screen below. To establish the desired axis units and labels, simply enter the desired values in the boxes. This allows you to examine (plot) small sections of experimental data and fitted curve. The Default button will calculate appropriate axis units based on the experimental data itself. 


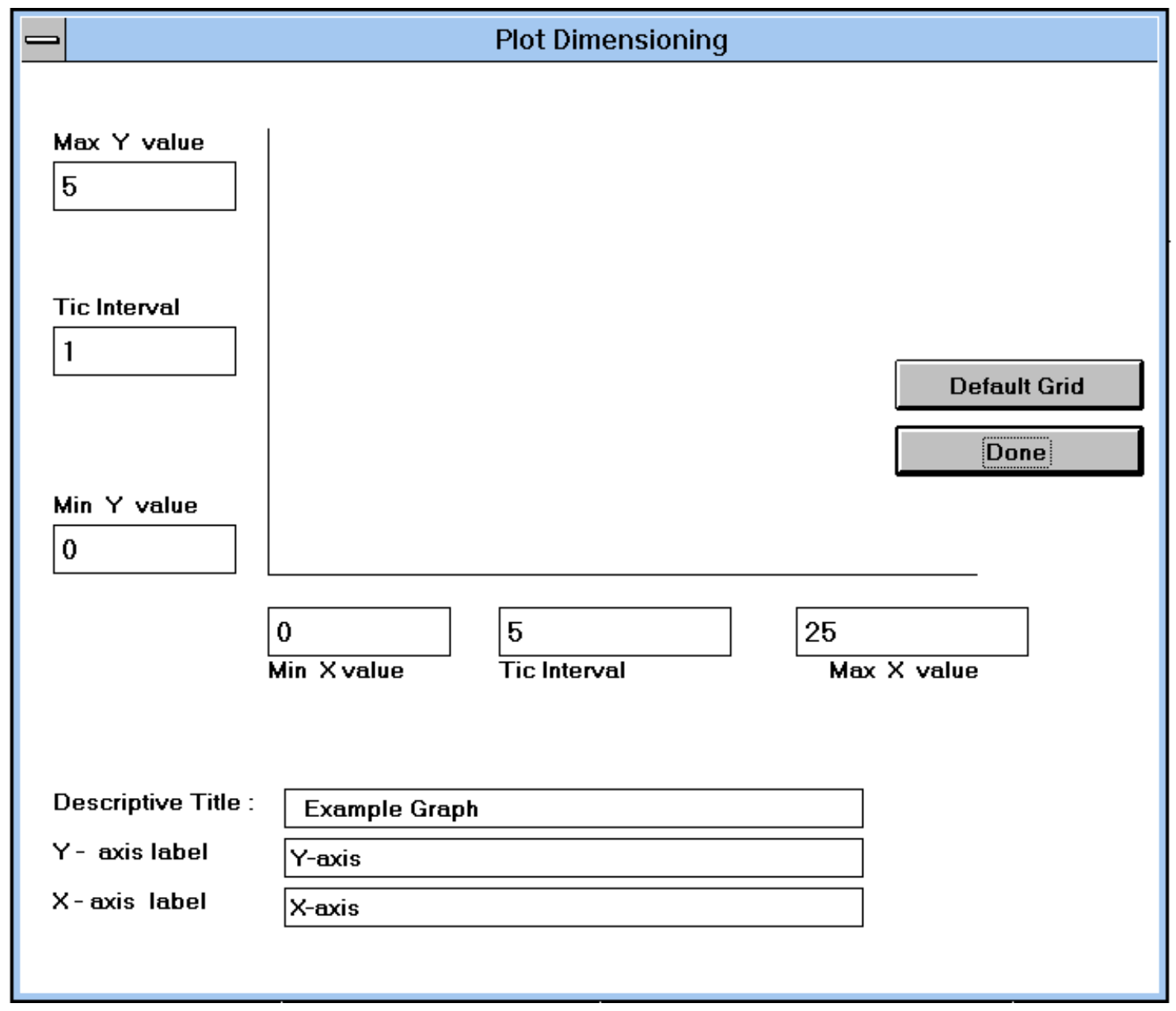

The Coefficients menu selection generates a display of the values calculated for each of the polynomial coefficients. For each data point, it lists the actual y-value of the data, the y-value estimated by the fitted polynomial, the residual and the percent difference between the actual and the estimate. At the bottom, the average value and the standard deviation of the residual is displayed.

The Example menu selection creates a set of "experimental data points." This option provides a method to allow exploration of the behavior of the various operations available with "Curvefit" without requiring the user to enter actual data. It is most useful is explaining the operation of "Curvefit."

The Colors menu selection allows the user to change the screen colors assigned to the various items and fields on the display screens. This is necessary because Microsoft Windows allows the user to tailor the colors applied to all applications. In some cases, user color specifications and/or specific video card options can result in color combinations which are unpleasant or difficult to see. The colors dialog provides complete flexibility in specifying colors. The user's pre-selected colors will be restored at Exit. 
The Print menu selection does just that. The graph is printed on one page and the information displayed by the Coefficients menu selection is printed on a second page. The printing is done on the Microsoft Windows default printer. If desired, the default printing parameters can be changed through Windows.

\section{Design Issues}

The principal design goals for the two programs were the same. Important goals included ease of initial installation, intuitive or self-guiding activation of the various available functions, conformance with the "look-and-feel" of Microsoft Windows applications, and accuracy of the function fitting and graphing operations.

The applications are distributed on 3.5" diskettes. The steps required to install the applications are simple enough that anyone who has used Microsoft Windows can expect to do it correctly the first time in under two minutes. Simply insert the first installation diskette in the A:I drive and run A:ISETUP. As the applications install themselves, they will prompt for some installation options. Most users should select the defaults and continue. At completion, the user will have a new program group with an icon to launch the application.

As shown in the Description \& Operation section, the various operations performed by the applications are readily accessible. In those cases where parameters are required to complete an operation, the data screens presented to collect the parameters are very easy to use. Also apparent is the conformance with the look-and-feel common throughout Microsoft Windows applications.

"Curvefit" determines the best-fit $\mathrm{n}^{\text {th }}$ degree polynomial by performing a least squares fit to the data.

"Diode" determines the best-fit of the data to an equation of the form: $i_{D}=I_{S}\left(e^{v_{D} / n V_{T}}-1\right)$ where $\mathrm{n}$ is a constant between 1 and 2 depending on manufacturing and $V_{T}=\frac{k T}{q}$

$\mathrm{k}=$ Boltzmann's constant $=1.38 * 10^{-23}$ joules $/$ Kelvin

$\mathrm{T}=$ the absolute temperature in Kelvin $=273+$ temperature in ${ }^{\circ} \mathrm{C}$.

$\mathrm{q}=$ the magnitude of electronic charge $=1.602 \times 10^{-19}$ coulomb

\section{Tests}

The curve fitting algorithm was evaluated in comparison to two other polynomial fitting programs. For several different sets of data points, a polynomial of each degree 1 to 5 was fitted using a statistical package available on our VAX computer, using MATLAB, and using Curvefit. The coefficients generated by each of the three packages were generally the same to 6 or 7 
significant figures. The polynomials generated by Curvefit generally gave average residuals with absolute value somewhat closer to zero than the other two.

\section{Questionnaire \& results}

The first use of Curvefit in the Materials Science Laboratory was evaluated by the students in the class. This course was their first exposure to Curvefit. The data measured and graphed was fitted with a straight line, the slope of which is Poisson's Ratio. Following their introductory use of Curvefit, they were asked to complete a questionnaire which solicited both critique of the extant system and suggestions for improvement. There were no significant gripes from the students. The most substantive suggestion was to replace the point-by-point data collection mechanism with a spreadsheet style collection mechanism. This is being evaluated. Also suggested was the addition of a Windows style help system.

\section{Conclusions}

We have observed that students are using the Curvefit program in their other courses to generate good quality graphs of measured quantities. Within the windows environment, they can generate a good looking graph with Curvefit and cut-and-paste it into their laboratory report (using Microsoft Word or a similar word processor).

\section{GLENN S. KOHNE}

Glenn S. Kohne is an Associate Professor of Electrical Engineering at Loyola College in Maryland. He received a B.S.E.E. from the University of Maryland in 1970. He worked as a Lead Electrical Engineer for Raytheon Company and as a Lieutenant Commander for the U.S. Public Health Service. He received a M.E.S. from Loyola College in 1981. He is currently in his $15^{\text {th }}$ year at Loyola. 